\title{
FIRST DISCUSSION SESSION
}

(Wednesday afternoon; 6 September, 1972)

(following review papers by Batten and Huang)

\section{Chairman: A. D. THACKERAY}

Thackeray: First we should express our thanks to Dr. Batten and Dr. Huang for the papers they have just presented. I've been asked to encourage as much freedom as possible in the discussion of these papers and not to limit ourselves to the order on the programme. Would anyone like to comment on any point?

Sahade: Could we ask Dr. Hall to present his ideas on SW Cyg?

Hall: Dr. Batten, in his review paper, talked about streams and disks, and clouds. In my analysis of the light curve of SW Cyg (Hall and Garrison, 1972), I found the need to hypothesize a different kind of feature, which I will call a lump. Perhaps I should not call it 'lump', but that is easier to say than 'protuberance' - the term used in the paper. Let me explain exactly why I found this idea necessary. Perhaps someone can suggest another way to explain the peculiar feature.

The binary SW Cyg is a rather typical Algol-like eclipsing binary, with an A2e primary and a $\mathrm{K} 0$ subgiant secondary which fills its Roche lobe. Primary eclipse is total, with totality lasting about $2.5 \mathrm{~h}$. The peculiarity which bothered me most was that totality was not exactly flat. This was true in the visual and in the blue and also in the ultra-violet, but there is something else wrong with the ultraviolet light-curve which I will discuss later. The light rises gradually, as you go from mid-eclipse to third contact, by something like $0^{\mathrm{m}} 05$. There is admittedly some scatter in my 1968 observations, because SW Cyg is quite faint at minimum, but this same effect shows up very nicely in Walter's 1964 observations and also in Wendell's 1900 visual obserservations. So there can be little doubt that this effect is real and at least semi-permanent. This same rise occurs in blue and ultra-violet, and the amount of the rise corresponds to the same percentage of the light of the hotter star. In other words, this increase has the colour of the hotter star, which makes me think that the cause is something that is actually part of the hotter star.

This is the way the lump model works. The lump effectively extends the photosphere of the hotter star by about $10 \%$ on its leading hemisphere. As the hotter star moves from mid-primary eclipse towards third contact, the lump will be the first part of it to emerge from behind the cooler star. Since the lump has about $10 \%$ of the 'radius' of the hotter star, it will have about $1 \%$ of the projected area. Thus, with the same surface brightness, it will account for about $1 \%$ of the light. This light, relative to the light of the cooler star visible throughout totality, can produce the extra brightness observed around third contact.

Several other peculiarities can be accounted for by this lump. First, Dr. Walter noticed in his light curve of SW Cyg that the descending branch was fainter than the ascending branch, at least the upper part of it was. This could be the eclipse of the 
lump. Second, Dr. Walter noticed dips in his light curve outside eclipse, where there should not have been any. In particular I notice the two depressions around phase $0^{P} .2$ and phase 0.8 . These two dips, if they were separated by exactly $180^{\circ}$, could be explained very nicely as the phases at which we see the hotter star end-on, (if the starplus-lump combination is thought of as an elongated star). The effect could then be analogous to the familiar ellipticity effect caused by tidal elongation, except that the elongation is not in the direction of the line of centers.

Whenever the descending branch is fainter than the ascending branch, as in the light curves of $\mathrm{U}$ Cep and RZ Sct, it is traditional to consider the faintness as an effect of absorption by the stream. But a stream cannot easily explain the other features. Between mid-primary eclipse and third contact the stream is behind the cooler star and cannot be used to explain peculiarities. Furthermore, a stream cannot conveniently explain the dips around 0.2 and 0.8 . Another problem is that the stream cannot easily explain a peculiarity which has the colour of the hotter star unless it absorbs non-selectively. Electron scattering is non-selective but, although this source of opacity can be operating in streams near B stars such as those in U Cep and RZ Sct, can there be electron scattering in the stream near the A2 star in SW Cyg?

The essential characteristic of the lump, I want to emphasize, is that its density is sufficiently large that it is optically thick in the continuum observed in the $V$ bandpass. Therefore the surface of the hotter star, as defined by its photosphere, is not spherical but actually has a bulge in it. Why the lump is there is another question. That is not my problem!

Another peculiarity shows up in the ultraviolet when we look at second contact. Second contact is quite well defined in $V$ and $B$, but in $U$ it is rounded, so much so as to give the impression that the eclipse is partial in $U$. At the phase of second contact, the light in $U$ is still about 0.3 above the level it eventually reaches at minimum. This light almost certainly comes from the stream or else from the region where the stream encounters the hotter star on its trailing side. Emission from the stream should come primarily in the Balmer continuum and therefore mostly in the $U$ but almost not at all in the $B$ or $V$.

McNamara: Your $B$ curve also exhibits a rounded edge at second contact, although it is not as pronounced as in the $U$ curve.

Hall: Dr. McNamara noticed in Figure 1 of my paper (Hall and Garrison, 1972) that a suggestion of the rounding around second contact appears in the blue, although not at all in the visual. Perhaps this might be the effect of including several of the Balmer lines in the $B$ bandpass, or perhaps the short wavelength tail of the $B$ bandpass reaches down far enough to include a little bit of the Balmer continuum.

Thackeray: Would Dr. Walter like to comment on this?

Walter: Let me first consider the problems of SW Cyg from a general point of view. In an Algol-type system with a period of about five days, like SW Cyg, the gas stream, which leaves the subgiant near the Lagrangian point, $\mathrm{L}_{1}$ can be made to intersect different parts of the surface of the bright star by quite small changes in its direction. For phases outside eclipse, one must take into account the effects on the 
light curve of absorption by the gas stream, and emission from regions where the particles in the stream strike the bright component. If the particles meet the surface of the bright star almost tangentially, as in AD Her (Korsch and Walter 1969), the photometric effects of the stream can only be seen in the part of the light curve between secondary and primary eclipses. The absorption is particularly strong immediately after secondary eclipse, when we are looking along the stream. If the particles arrive somewhere on the part of the following side of the bright component that faces the subgiant, this region of the surface will be brighter and can be seen without absorption immediately after primary eclipse. At this phase, the rectified light curves of many Algol-type systems show a hump. The light curve of $U$ Cep shows a large hump - smaller ones are seen in the light curves of SW Cyg and TT Lyr (Walter, 1971a). The light curve of SW Cyg shows this asymmetric gas-stream effect clearly (Walter, 1971b).

My observations of SW Cyg, obtained in 1964 in Sicily, were not numerous enough to conclude any more from the light curve outside eclipses. The observations of the eclipse phases, made in $B$ and $V$, were good and seemed to be suitable for a determination of the photometric elements. For a long time, however, although I tried hard, I could not obtain a satisfactory solution in both colours with the same values for the ratio of the radii, $k$, and the orbital inclination, $i$; but, after many vain attempts, I found that solutions with quite usual limb-darkening coefficients fitted the light curves very well, in the neighbourhood of total eclipse.

I then abandoned the usual Russell-Merrill method, in which the form of the eclipse curve is used to derive the photometric elements. If a light curve is suspected to be distorted by luminous regions (on the surface of one of the stars), the geometric parameters must be obtained from data which are independent of the intensity distribution over the stellar surface. For SW Cyg, the phase of second contact, and the depths of the minima were used. Because the primary eclipse is asymmetrical, I assumed an extinction by the gas stream of two per cent in the descending branch.

In this way, I obtained good representations of the central parts of primary eclipse, using exactly the same values of $k$ and $i$ in both colours. In the outer parts of the the eclipse, however, a residual excess luminosity of about two per cent remained, in both colours. This can be interpreted as additional light from hot regions which happen to be eclipsed within the primary eclipse. The known geometry of the eclipse of the stars allows us to locate these hot regions on the bright component. In the case of SW Cyg, the surprising result was that these regions cannot be located near the equator of the bright star; they must be situated in high latitudes.

The old, very good observations of SW Cyg made with the polarizing photometer by Wendell can be represented in a quite similar manner, if exactly the same geometrical elements are used as were obtained from my $V$ observations. Probably there was rather more additional light in 1900 . The asymmetry of the primary eclipse was also larger then, and I adopted an extinction of three per cent.

The fact that the luminous regions are at high latitudes suggests the action of magnetic forces which compel the ionized particles of the gas stream to move out of the 
orbital plane. If there are magnetic fields, either on the bright component, or between the components, where the gas stream flows, then such a binary may beconsidered to work like a big mass spectrograph. If there are magnetic fields, some particles are strongly influenced by them, follow the lines of force, and arrive at high latitudes. Other particles may be more strongly influenced by gravitational forces and arrive near the equator of the bright star. In my report at the I.A.U. Colloquium No. 16 (Walter, 1971a) I distinguished between $g$ (gravitational) and $m$ (magnetic) regions for hot spots. So I think my observations and Dr. Hall's do not contradict each other, but concern complementary aspects of hot-spot phenomena.

Biermann: I would like to comment just very briefly on this. What you expect from the theoretical calculations is a gas stream leaving the Lagrangian point and expanding a bit (depending on the mass ratio of the system and the angular momentum per unit mass). You get some sort of hot spot that is a standing shock where the stream meets the star, and another standing shock where the part of the stream that goes around the star meets the directly expanding stream. Are Dr. Hall's observations consistent with the hypothesis that his 'lump' is a kind of weak shock between the two streams?

Hall: I think of the lump more as a significant accumulation of matter of sufficiently high density to be optically thick in the continuum. The colour of the lump seems to be different from that of the stream. The stream emits almost entirely in the ultraviolet, whereas the lump has the colour of the hotter star.

Biermann: I can't propose any detailed model for the shock. I would expect the stream by itself to have very different characteristics from a shock...:

Hall: What would you predict the colour of the shock to be?

Biermann: Oh, I wouldn't predict any colour.

Plavec: I think we might try to get at some prediction of the colour. It's surprising that the colour should be the same as that of an A-type star because when the stream falls on the star and creates a hot spot, if its kinetic energy is very rapidly converted into energy of thermal motion, we can write that the maximum possible temperature would be $1.5 \times 10^{7} \mathrm{~m} / R$ (where $m$ and $R$ are the mass and radius of the star, respectively, in solar units). Now, for an A3 star, $m / R$ is approximately unity, and you have a very high temperature. Even $X$-rays could be produced. Of course, we are not considering the problem of intensity, but certainly the spot would be really hot. When the material falls at a high speed onto the star, even if you assume that the efficiency of the process is very low (say one per cent) you still get a very high temperature-certainly nothing like the surface temperature of an A-type star. So the bulge in Dr. Hall's picture is, first, very difficult to explain dynamically - in the direction he wants it - and second, its colour index indicating (if it is measured accurately enough) so low a temperature is also difficult to explain. Perhaps Dr. Biermann's suggestions might save the picture proposed by Dr. Hall, if two streams are meeting - but they may meet rather far from the surface of the star, and the velocities will be so terribly high.

Underhill: This brings up the problem of detecting a gas stream around a hot star. To detect the gas stream you must observe at a wavelength in which the stream is 
emitting strongly. If we have a spot with a temperature in the range of one to ten million degrees, the spot will radiate very strongly at wavelengths shorter than $2000 \AA$. This means you must observe from above the Earth's atmosphere using either a photometer behind a small telescope or a spectrometer. However, at present there is no instrument up in space capable of observing faint stars. OAO-2 does have photometers but they can be used only on stars brighter than about 6th magnitude. I don't know how faint SW Cyg is, but I suspect it is too faint for OAO-2. There is a lot of work to be done in photometry in the far UV but first of all we have to get aloft an instrument capable of doing photometry on faint objects. A second goal is to launch an instrument capable of doing spectrographic work. At present there is one instrument planned for purely spectrographic work in addition to the two OAO's which are now flying. If the gas in the streams or rings Batten was telling us about has a temperature between $10000^{\circ}$ and $30000^{\circ}$, it may be detected by observing the Balmer lines of hydrogen, which can be done from the ground. If the temperature is in the range $5000^{\circ}$ to $10000^{\circ}$, the emission in the Balmer lines will be weak and the gas may be visible in the resonance lines of $\mathrm{Ca}$ II or better still in the resonance lines of $\mathrm{Mg}$ II at $2800 \AA$. To observe these requires a satellite-borne instrument. Thus we see that space astronomy has much to offer to the study of binary stars and of the gas streams in binary systems, for it will permit the detection of very hot streams and spots as well as cool streams of gas.

Smak: The restricted three-body approach, used to compute the particles trajectories, was surprisingly successful in explaining several properties of the circumstellar matter. And, while it is obvious that there are regions of higher density where this approach cannot be correct, I believe that - for reasons of simplicity - we should go with it as far as possible trying to interpret at least some of those many problems which are not yet explained.

Before I shall discuss one such problem, let me mention once again a few trivial facts. Suppose the secondary component loses matter through the inner Lagrangian point. The stream of matter goes toward the primary component and - if there is no circumstellar matter - it lands on the surface of the star (Figure la). If the amount of matter is small, it can be accumulated by the star. If its amount is large, however, and particularly - if the amount of momentum it carries is large, then a rotating disk must be formed. The mean radius of such a disk can be estimated from the angular momentum considerations and this is usually done under the assumption that other momentum transfer processes can be neglected. With the disk being present, the stream of matter from the secondary component must collide with its outer portions and a hot spot must be formed at the place of collision (Figure 1b). This is what is typically observed in systems of the nova and U Gem type. It may be added that usually the amount of momentum carried by the stream is so large that the radius of the disk must also be large and - as a rule - the spot must be formed in a relatively narrow range of phase angles roughly in a position indicated in our schematic picture.

Let us assume, however, that the outer dimensions of the disk are not always the same. Indeed, we know already examples of variable dimensions of the disk, like 


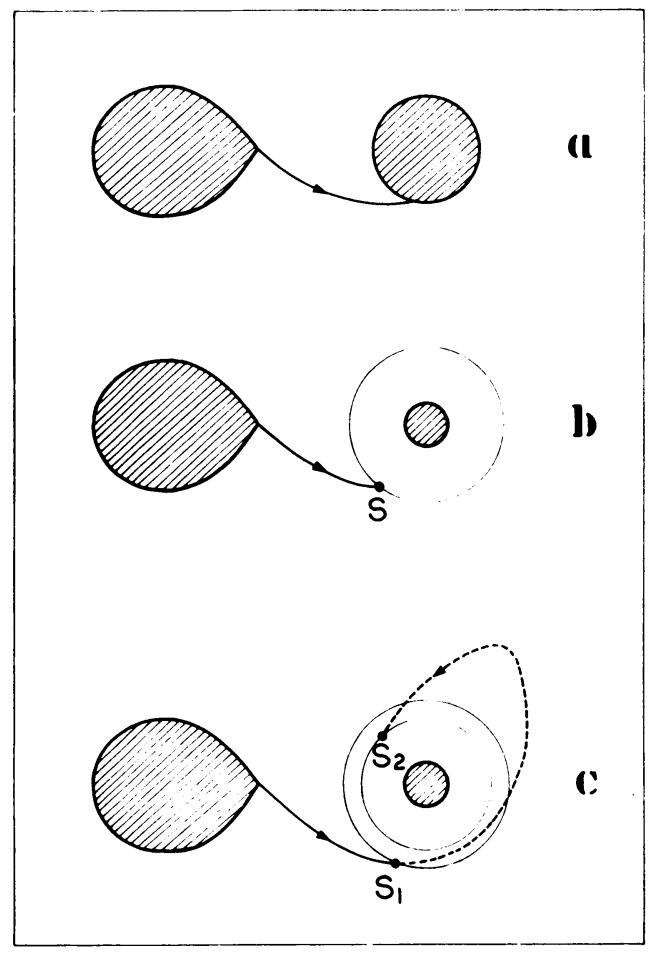

Fig. 1. Trajectories in U Gem systems.

U Gem or RW Per. Such phenomena are connected presumably with the variable rate of the momentum transfer from the disk to the primary component or to the orbital momentum and/or with some other instabilities occurring within the disk. Let us further assume that - at one time - the disk can become much smaller. Then the stream of matter can go around the primary component and collide with the disk at an almost opposite phase angle. Figure lc shows two situations corresponding to the two different radii of the disk with the two resulting locations of the hot spot, S1 and S2. If we let the radius of the disk vary continuously, we get two ranges for S1 and $\mathrm{S} 2$, respectively. Detailed calculations would probably show that - for any given system - both these ranges are rather narrow. However, with the stream particles travelling so close to the edge of the disk, we should consider here the effect of slowing them down by the lower density medium extending far beyond the conventional limits of the disk. With this effect taken into account we could probably obtain nearly any location for the hot spot although it is clear that such a spot may no longer be a distinct, point-like feature.

These considerations, if correct, may offer an explanation for the so-called periastron, or O'Connell effect including the cases where this effect is variable. Indeed, a hot spot located at an arbitrary phase angle can produce an arbitrary distortion of the photometric curve. Its variable location and variable intensity of its radiation 
can account for the variable light curves. Of course, as long as we do not know too much about the behaviour of the disks, we cannot insist on applying this explanation to all cases but at least in some of them this mechanism may be more plausible than the others.

Finally, I wish to point out that the situation presented in Figure 1c may, in fact, occur in certain Algol-type systems. I refer to the extensive distortions in the radial velocity curves which have been successfully interpreted in terms of the streams of gas circulating nearly around the primary component; in contrast to the disks, they produce absorption lines and are less regular.

R. E. Wilson: I'd like to comment that the temperature Dr. Plavec suggested is probably too large by considerably more than one order of magnitude. By way of illustration, if one does a similar calculation for very compact objects - white dwarfs or neutron stars - one finds temperatures of $10^{9}$ to $10^{12}$ degrees, but the observed temperatures of typical X-ray sources are, of course, only about $10^{7}$ degrees. The temperature obtained from the formula is up to five orders of magnitude too high, and it is, indeed, a very extreme upper limit. Normally temperature calculations involve a balance between heating and cooling, but the temperature quoted by Dr. Plavec corresponds to all heating and no cooling. As the material is heated it begins to reradiate the energy it has received and immediately cools. Furthermore, the material coming in shares its thermal energy with the material of the star, so I think temperatures of the order of $3 \times 10^{7} \mathrm{~K}$ will not be encountered at all in this kind of situation.

Plavec: No doubt this is an upper limit, but I doubt if it is off by, say, three orders of magnitude. We will probably have discussion on X-ray sources later. I wanted to comment actually on Smak's picture. It's true that in certain cases (mostly in those in which he is interested) the primary component is so small that the ring forms and then the hot spot is situated mainly on the outside of the ring. But, unfortunately, in the Algol systems, which are of relatively short period, the radius of the primary component is about 0.2 (expressed in units of the orbital radius) which means, if you perform the calculations Smak referred to, that the stream will hit this component directly on its first approach. It is very difficult to escape from this fact, and I think Dr. ;Batten will agree that our idea now is, that in the case of U Cep and U Sge, the ring or disk we observe may actually be due to some scattering of particles by the primary, after impact. In any case, the ring will probably not be dense. Hot spots on the ring can form in binaries where the primary component is very much smaller - smaller, for example, than 0.1 . This easily happens in systems with periods of about 200 days, or in systems containing degenerate stars (where the radius of the star itself is quite small). Then you can look for a possible hot spot on the ring. But generally in Algol systems like SW Cyg, I think we must assume that most of the material falls on the star just because of its larger size.

Huang: Most of you talk about the hot spot on the ring. If you have a gas that hits on an extended medium, you produce a hot spot - but the ring is tenuous. Why do you talk about a hot spot on the ring? If you have a ring maintained only by angular 
momentum with no elasticity, the stream of gas will destroy the ring instead of having a shock or anything.

Smak: : Even with densities in the disk of the order of $10^{12}-10^{13}$ particles $\mathrm{cm}^{-3}$, which I believe is an underestimate, one finds that the mean free path of the stream particles is one or two orders of magnitude smaller than the disk's radius. So, the collision must effectively take place in the outermost part of the disk.

Underhill: I am concerned about the estimates of density in these rings or streams. The average particle density in a late B type star or an A type atmosphere is $10^{13}$ to $10^{14}$ particles per $\mathrm{cm}^{-3}$. In supergiant atmospheres the density is about $10^{12}$. If you have coronal conditions the density is about $10^{7}$; when forbidden lines appear the density is $10^{4}$ to $10^{7}$. Now some of the densities Batten quoted were $10^{16}$ to $10^{17}$ particles $\mathrm{cm}^{-3}$ and I heard Smak say he would like them an order of magnitude greater. I don't understand it. You cannot have such large densities and obtain the spectroscopic observations which you have. Even in a dense main-sequence star like the Sun the density in the atmosphere is not much greater than $10^{15}$ particles per cubic $\mathrm{cm}$. It cannot be an order of magnitude greater in a gas stream from a $\mathrm{K}$ type giant.

Batten: I think that I have quoted densities as high as $10^{16}$ or $10^{17}$ particles $\mathrm{cm}^{-3}$ only in order to criticize them. I don't think they are that high myself. I do think they may be as high as $10^{13}$ particles $\mathrm{cm}^{-3}$ because, after all, at least in the case of Algol systems, the streams originate in stellar atmospheres of about that density. Forbidden lines, of course, must come from regions of lower density - as I pointed out in my review. You must distinguish between regions of higher density (which I call streams and disks) and regions of low density (which I call clouds). Even if you don't like my terminology, I have made the distinction.

McNamara: When Batten discovered the emission lines in the spectrum of $U$ Cep, I think he mentioned that their detection depended very critically on when the plates were exposed (in relation to the contacts of the eclipse). Might it not be that the line intensities are changing quite dramatically because of real changes in the disk?

Batten: You've quoted me correctly but I've changed my mind about this. I still think that timing of exposures may be important, but, just about a year ago, $\mathrm{Mr}$. Baldwin and I made simultaneous photometric and spectroscopic observations during eclipses of U Cep. We know that we began and ended our exposures within a few minutes of second or third contacts, and we are reasonably confident that if emission had been detectable we would have detected it again. We did not. Therefore we believe that either the disk itself, or the state of excitation of matter within the disk really" does change.

Hall: I would like to ask Dr. Batten if he remembers a relatively old paper on U Cep by Miczaika (1953), in which he hypothesized a lump in U Cep to explain the same sort of photometric peculiarities in his light curve which caused me to hypothesize a lump in SW Cyg. Let me point out that Miczaika's model referred to his yellow-light curve, which should have been relatively unaffected by emission lines per se, and furthermore that his lump had the same orientation: on the leading side of the hotter 
star. In both U Cep and SW Cyg the first part of the descending branch of primary eclipse is relatively too faint (caused, I would say, by the eclipse of the lump by the cooler star). In both systems there is an increase in light during totality as you go from mid-eclipse to third contact (caused, I would say, by the emergence of the lump from behind the cooler star before the hotter star itself emerges). And there are two dips in the light curve outside eclipse approximately $180^{\circ}$ apart from each other (caused, I think, by the fact that at these phases we see the elongated star-plus-lump end-on). I wanted to ask Dr. Batten to what extent he considered Miczaika's model believable, at least in the case of $U$ Cep.

Batten: Yes, I remember that paper. I believe the light curve of U Cep that Dr. Walter showed us was Miczaika's. My own feeling is that the way in which Miczaika made up his normal points concealed the true nature of the variation outside eclipse. As I mentioned in my review, most light curves of $U$ Cep show a sudden dip of about $0^{m} .1$ somewhere between $0^{P} .8$ and 0.9 in phase. This feature is seen very clearly in light curves of different colours, even an infrared light curve obtained by Khozov and Minaev (1969) at $8100 \AA$ shows it quite clearly. I think your light curve, Dr. Catalano, shows it?

Catalano: Yes, the light curve we have in Catania shows a change near phase 0.8 of about 0 . 1 . I should like to remark that the light change occurs just at the same phase as the radial-velocity curve shows a jump.

Batten: Miczaika's light curve is the only exception. I believe the distribution of his observations, and the way he combined them to form his normal points, have produced a misleading picture of the out-of-eclipse light variation, and for this reason I am a little skeptical of his model.

Hall: Miczaika found that the duration of totality was variable from cycle to cycle. At third contact he found that the exact phase at which the light suddenly increased was significantly different from one cycle to the next, as if the photosphere of the leading edge of the hotter star was at a different place at different cycles.

Batten: There is evidence for real variation in the light of the secondary star, and different light curves show quite different sorts of variation during totality.

Thackeray: How sudden is the drop in brightness at phase 0.8 ?

Batten: Quite sudden. At most it takes a few hundredths of a period, I believe.

Catalano: Yes, it is quite steep, but I don't remember now precisely how long it takes.

Underhill: I would like to ask Dr. Huang whether the decreasing percentage of stars with shells through types B and A shown in his table is real, or whether it is a result of the difficulty of detecting a shell at type $A$.

Huang: There are few A-type stars with rings. I believe it is an intrinsic deficiency. Since emission lines are found in the spectra of binaries with A-type primaries, it appears that A-type stars have temperatures high enough to excite the gas in the ring. Consequently the deficiency of gaseous rings about rapidly rotating A-type stars is intrinsic.

Underhill: If we had other criteria to rely on than the hydrogen line, for the detection of these shells, would you say that we could see more shells? 
Huang: I am not sure of any other criterion that could be used to detect a rotating shell that is found by the hydrogen lines.

Underhill: There is nothing physically to prevent the cooler stars from having shells that produce emission lines. These cool shells would not emit in hydrogen, therefore we have not detected them that way, but is there any reason why there should not be extended atmospheres around late B-type and early A-type stars, similar to those around early B-type stars?

Huang: It does not appear physically that there should be a ring around A-type stars, and we don't know whether or not the extended atmospheres you have mentioned exist. But around stars with rings, there must also be another substratum, as I mentioned in my talk, because the ejected matter cannot all go into the ring. Some will be left there as an envelope that is distinct from the ring. I take the view that A-type stars have no envelope or ring, B-type stars have rings, and most O-type stars that show emission in their spectra do not possess rings.

Underhill: I don't think I agree with you....

Huang: Yes, of course. This is why at the beginning of my talk I emphasized that everything can happen. I used the phrase 'my present understanding' because I may change my mind tomorrow. Therefore I do not dispute your disagreement!

Biermann: I was thinking of the possibility of rings in single A-type stars and the question of those A-type stars in binaries, that show emission from their disks. How can you be sure that the emission is caused by the A-type star and not something else?

Huang: Because the other component star is of an even later spectral type and cannot be responsible for the excitation.

Biermann: But are you sure that the emission is not a feature of the gas stream itself? When you have an oblique shock, strong emission could be caused by it. Therefore, no emission observed in the spectra of single A-type stars does not rule out the possible existence of rings.

Huang: Oh, you can always have many possibiiities, and in astrophysics there is no sure thing. You can make all kinds of assumptions. I wouldn't rule out any other possibility. I tried to describe the excitation problem purely from the observational point of view, and used the component stars with rings, in binaries, as a model for comparison. Of course, I completely agree that this comparison may be inadequate; but if you introduce other possibilities there would be no end to it. The excitation is not a settled problem.

Smak: I think the case of rings in binary systems is quite instructive. We observe emission in the spectra of very few B-type stars and from very many A-type stars even of quite a number of F-type stars. (I refer to the spectral types of the primary components which are surrounded by disks.) We cannot blame the central A-type and F-type stars for the ionization of their disks. An even more convincing argument is provided by many novae, and U Gem type systems, in which the disks are optically thick so that the radiation from the very faint stars inside cannot penetrate the disk at all. So there must be another source of energy and I think we must agree that the ionization and excitation is of the collisional type with the kinetic energy being supplied 
by the stream coming from the secondary. Now, to return to the disks of single B-type and A-type stars, one can speculate that they are formed not only in the Be stars, but also in the A-type stars, except that the emission lines are not visible in the latter case because the ionization conditions are different. In addition to the different temperatures of stars, it may also be important to note that the amount of kinetic energy is different. If rotational break-up is, at least partly, responsible for disk formation, much larger velocities are observed in B-type stars. Therefore the ring of a B-type star can be bright and visible, and the ring of an A-type star may remain practically invisible, and the presence of absence of emission lines of hydrogen is not a definite test of the existence of a ring. We need another way of detecting rings.

Popper: I agree with Dr. Huang that it is not desirable to introduce unnecessary complications. But I also agree with Dr. Smak that the spectra of rings around A-type and F-type primaries are not different from those around hotter primaries in their

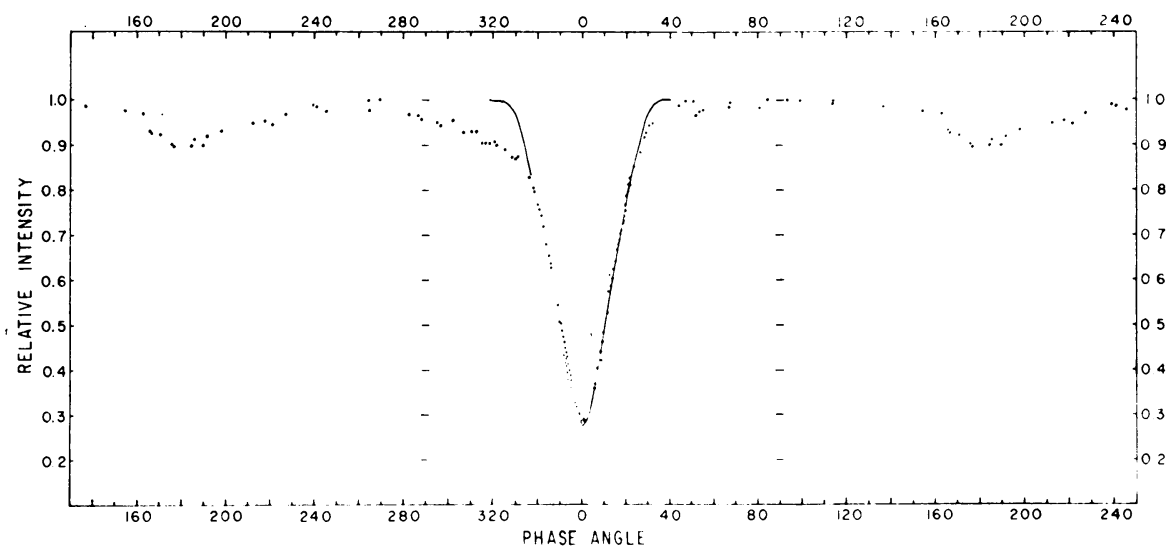

Fig. 2. The $V$ light curve of RZ Sct. The plotted data are normal points except for the smaller dots near the bottom of primary minimum which are individual observations. Redetermination of the period of the system, since this figure was drafted, has reduced the scatter of these latter points.

The solid line represents a preliminary solution. A more definitive solution is in progress.

degree of excitation, which is higher than that of the photosphere of these later types. There is pretty strong evidence of non-thermal effects. The spectra of the rings around the F-type primaries of KU Cyg and RZ Oph, in addition to the hydrogen and calcium emission lines, show an ultra-violet continuum. This continuum is not a Balmer continuum since it extends strongly to longer wavelengths.

Hansen: First, I'd like to remind you of the light curve of U Cep which Dr. Batten showed earlier (Figure 2 on p. 6). Notice the break in the curve, which he has already pointed out, that is seen just before primary minimum when there is a definite decrease in light before the beginning of the actual eclipse. There is also a noticeable slope upward at the bottom of the primary eclipse between second and third contacts. But notice also that the secondary eclipse is not symmetrical, the ascending branch of this eclipse is much less steep than is the descending one. This was not pointed out earlier. 
Dr. Hall has proposed a 'lump' model of the hotter star to explain the distortions in the primary eclipse, and I agree that such a model can explain these two features, but it does not explain the distortion of the secondary eclipse. Now look at the light curve of RZ Sct (Figure 2). Beginning at the left of the diagram, we notice that the secondary eclipse shows a relatively steep drop into eclipse and a much more gradual rise coming out. In the primary eclipse there is a long, gradual slope going into eclipse while the up-branch is steep all the way up, and the shoulder at the end of the eclipse is much more distinct.

I would like to ask how Dr. Hall would explain this feature of the secondary eclipse, the depressed ascending branch, in terms of his model? It seems clear to me that the system of RZ Sct has some sort of mass concentration, a stream or an eddy such as has been previously proposed, that is located on the side of the system corresponding to the advancing side of the secondary. Material in this position could cause an absorption during the approach to primary eclipse and also as the system is emerging from secondary eclipse. So the interesting thing to me is that RZ Sct has the same feature in the secondary minimum that is seen in U Cep, and I wonder how Dr. Hall's 'lump' model might explain this feature.

Hall: To explain it with the 'lump model', we would have to place the bright lump so that just after secondary eclipse it would be on the far side of the primary star, hidden from the observer. There should then be a minimum in the light curve because the lump is invisible (leaving the eclipses themselves out of account). This position for the lump is also the one needed if the loss of light before the beginning of primary eclipse is to be interpreted as an eclipse of the lump by the cooler star. If the lump is in this position, there should also be another minimum in the light curve, $180^{\circ}$ in phase from the first, namely right after primary eclipse. At that phase the star-pluslump (considered as an elongated star) would be seen 'end-on'. Your observed points do fall below your computed curve at fourth contact (as well as at first contact). You will have to tell me if these residuals can be interpreted as the result of such a minimum in the light curve.

There may be one difficulty with your interpretation of the dip after secondary eclipse as an effect of absorption by the stream and eddy. I think you said that the cooler star accounts for only ten per cent of the total light of the system, and the dip after secondary eclipse is about $0^{\mathrm{m}} \mathrm{l}$. If so, then the stream and eddy must absorb virtually all of the light of the cooler star.

Hansen: The cooler star does account for about $10 \%$ or $12 \%$ of the total light of the system, but the 0.1 that you refer to is the maximum depth of the secondary eclipse.

If in the secondary eclipse we compare the ascending branch, which is depressed, with the descending branch we find that the extra loss of light is, at most, about $35 \%$, considering the amount that is lost at the midpoint of the secondary eclipse as $100 \%$. A similar comparison in the primary eclipse shows that the stream absorption amounts to about $10 \%$. It is true that to absorb about one third of the light of the cooler star requires a large, dense stream, but the spectroscopic evidence also indicates that RZ Sct does in fact possess an extensive stream and disk system of some sort. 
One further comment might be of interest. The two halves of the light curve of RZ Sct look as if they might belong to two different systems. From primary minimum to secondary minimum it looks like the light curve of a typical Algol system, but from secondary minimum to primary minimum it looks more like the curve of a system such as $\beta$ Lyr.

Batten: I have tried to explain the asymmetry of the secondary eclipse of U Cep in terms of a bright spot near the Lagrangian point of the secondary component, where the mass is actually ejected from the secondary star. I'm not very happy with the explanation but it does fit, qualitatively. Dr. Fracastoro has compiled an Atlas of light curves, and it is clear from this that asymmetric secondary eclipses are very common and we certainly must try to explain why this is so.

Devinney: I am surprised that Dr. Hall did not mention HS Her $(\mathrm{B} 4+\mathrm{A} 4)$ in this connection. The rise to maximum following mid-secondary eclipse is too slow, in this system, giving the impression that the star recovers too slowly from the eclipse. In addition, the 'flat' secondary minimum siopes upwards. On the other hand, from the phases of contacts alone, we can obtain very good approximations of the fractional radii of this two-spectra system, and we find that both stars lie well within their Roche lobes. Thus it seems difficult to blame gas streaming for the peculiarity. I've found that a star spot covering ten per cent of the primary's disk, and having a temperature of $12000 \mathrm{~K}$ satisfies the light and colour curves.

Hall: I really should have thought to mention HS Her myself, but thank you for bringing it into the discussion. In this system we have a B4V primary and an A4V secondary. The major complication occurs right after secondary minimum. It is as if the system refuses to come out of secondary eclipse. Full light is not recovered until almost 0.1 after where fourth contract should have been. This effect shows up most strongly in $U$ and less strongly in $B$ and $V$. I have described the system elsewhere (Hall and Hubbard 1971). The real puzzle is not the dip itself but that it occurs in a binary composed of two stars on the main sequence, both well within their Roche lobes and apparently perfectly normal. As I understand the situation, there is absolutely no good reason why there should be photometric complications in a system like HS Her.

Thackeray: It has been suggested that we adjourn this meeting until tomorrow morning.

\section{References}

Hall, D. S. and Garrison, L. M.: 1972, Publ. Astron. Soc. Pacific 84, 552.

Hall, D. S. and Hubbard, G. S.: 1971, Publ. Astron. Soc. Pacific 83, 459.

Khozov, G. V. and Minaev, N. A.: 1969, Trudy Astr. Obs., Univ. Leningr. Gos. $26,55$.

Korsch, D. and Walter, K.: 1969, Astron. Nachr. 291, 231.

Miczaika, G. R.: 1953, Z. Astrophys. 33, 1.

Walter, K.: 1971a, IAU Colloquium No. 16 (in press).

Walter, K.: 1971b, Astron. Astrophys. 13, 249. 